\title{
The Jidong Eye Cohort Study: objectives, design, and baseline characteristics
}

\author{
Kai Yang ${ }^{1,2+}$, Lele Cui ${ }^{1,2+}$, Guoyun Zhang ${ }^{1,2 \dagger}$, Xianwei Wang ${ }^{1,2}$, Xiaoxuan Zhu ${ }^{1,2}$, Yunfan Xiao ${ }^{1,2}$, Binbin Su ${ }^{1,2}$, \\ Daiyu Song ${ }^{3}$, Xinyao Zhang ${ }^{1,2}$, Yang Zheng ${ }^{1,2}$, Fan $\mathrm{Lu}^{1,2^{*}}$, Jia $\mathrm{Qu}^{1,2^{*}}$ and Ming $\mathrm{Li}^{1,2^{*}}$
}

\begin{abstract}
Background: To describe the objective, design and baseline characteristics of the Jidong Eye Cohort Study (JECS), a community-based cohort in China based on etiology, imaging and biomarkers. The JECS will clarify the pathogenesis of visual impairment and status of ocular indicators in the occurrence and progression of cardiocerebrovascular and neurological diseases.
\end{abstract}

Methods: Between August 2019 and January 2020, the JECS recruited consecutive participants aged 18 years and older from the Jidong communities in China. The demographic and clinical characteristics were collected by trained site personnel via face-to-face interviews. The relevant biological samples were also collected. The participants underwent comprehensive ophthalmic examination, such as retinal photography and optical coherence tomography (OCT) angiography. The following outcomes were measured annually: ocular vascular abnormality, optic nerve degeneration, cardiovascular diseases (CVD) and neurological diseases. The study will be performed until 2024.

Results: Among 3377 participants, the average age was $45.0 \pm 12.5$ years and 1809 (53.6\%) were women. Hypertension occurred in 825 individuals (25.0\%), diabetes in 258 (7.7\%), hyperglycemia in 474 (14.2\%), and a CVD history in 100 (3.0\%). The mean best-corrected visual acuity was 0.1 logMAR in the recruited subjects. The average OCT signal index was $8.2 \pm 1.2$. Additionally, the mean vessel densities for the entire measured area were $46.4 \%$ and $50.8 \%$ for the superficial and deep vascular complex, respectively. Mean area and perimeter of foveal avascular zone was $0.3 \mathrm{~mm}^{2}$ and $2.3 \mathrm{~mm}$.

Conclusions: The JECS is a large community-based prospective cohort in North China. Rich data collected from this study will provide the opportunity to identify risk factors, imaging, and biomarkers of visual impairment (either ocular vascular anomalies or optic nerve degeneration) and to evaluate their associations with CVD and neurological diseases.

Keywords: Cohort, Risk factor, Visual impairment, Cardiovascular disease, Neurological disease, Epidemiology

\footnotetext{
*Correspondence: lufan@eye.ac.cn; jia.qu@163.com; Im@eye.ac.cn

${ }^{\dagger}$ Kai Yang, Lele Cui and Guoyun Zhang contributed equally to this work.

${ }^{1}$ Eye Hospital and School of Ophthalmology and Optometry, Wenzhou

Medical University, Wenzhou, Zhejiang, China

Full list of author information is available at the end of the article
}

(c) The Author(s). 2020 Open Access This article is licensed under a Creative Commons Attribution 4.0 International License, which permits use, sharing, adaptation, distribution and reproduction in any medium or format, as long as you give appropriate credit to the original author(s) and the source, provide a link to the Creative Commons licence, and indicate if changes were made. The images or other third party material in this article are included in the article's Creative Commons licence, unless indicated otherwise in a credit line to the material. If material is not included in the article's Creative Commons licence and your intended use is not permitted by statutory regulation or exceeds the permitted use, you will need to obtain permission directly from the copyright holder. To view a copy of this licence, visit http://creativecommons.org/licenses/by/4.0/ The Creative Commons Public Domain Dedication waiver (http://creativecommons.org/publicdomain/zero/1.0/) applies to the data made available in this article, unless otherwise stated in a credit line to the data. 


\section{Background}

Approximately 217 million individuals around the world had moderate to severe visual impairment in 2015, among whom 36 million are blind and approximately $80 \%$ reside in developing countries [1]. About $82 \%$ of individuals with blindness and $65 \%$ with mild to severe vision loss are aged 50 years or older [2]. Retinal and choroidal vascular abnormalities, such as diabetic retinopathy, retinal vein occlusion and age-related macular degeneration, are the most common causes of irreversible visual impairment. Optic nerve degeneration is also regarded as a cause of visual impairment [2-8]. Therefore, it is critical to identify risk factors of visual impairment to prevent occurrence of ocular vascular abnormalities and optic nerve degeneration among middle-aged or older individuals with the use of images and biomarkers [9-11].

Patients with ocular vascular abnormalities often develop cardiovascular diseases (CVD) due to the comorbidities of diabetes, hypertension and hyperlipidemia. According to the Global Burden of Disease Study, ischemic cardiovascular disease, including stroke, is the major leading cause of death [12, 13]. Additionally, cognitive impairment is a common issue worldwide with an estimated 75.6 million individuals developing dementia by 2030 and approximately $\$ 604$ billion in social costs per year $[14,15]$. Thus, identifying novel risk factors for vascular abnormalities and nerve degeneration is critical to improve the screening of CVD and cognitive impairment in the early stages. The pathological change in the cerebral vasculature could be reflected in the retinal blood vessel due to their similar anatomy and physiology [16]. Given that retinal blood vessels are more easily and directly assessed, ocular vascular abnormality is being considered a prediction tool for the development of cerebrovascular disease [16-20].

Taken together, this study aims to evaluate the pathogenesis of visual impairment, including retinal vascular abnormality and optic nerve degeneration, and explore its association with cardiovascular or neurological disease in a community-based population. In this report, we describe the objective, study design, baseline characteristics, and strengths and potential limitations of the Jidong Eye Cohort Study (JECS).

\section{Methods}

\section{Study design}

The Jidong Eye Cohort Study (JECS) is a communitybased, prospective, long-term follow-up observational study conducted by the Affiliated Eye Hospital of Wenzhou Medical University. The JECS was designed to identify the etiology, imaging and biomarkers of visual impairment and its subtypes, evaluate the associations of ophthalmic lesions with the risks of cardiovascular and neurological diseases, and facilitate the identification and evaluation of vascular abnormalities and nerve degeneration in the early phase.

The study was performed in the Jidong community of Tangshan city in Hebei province. The location of the study is illustrated in Fig. 1. The study complied with

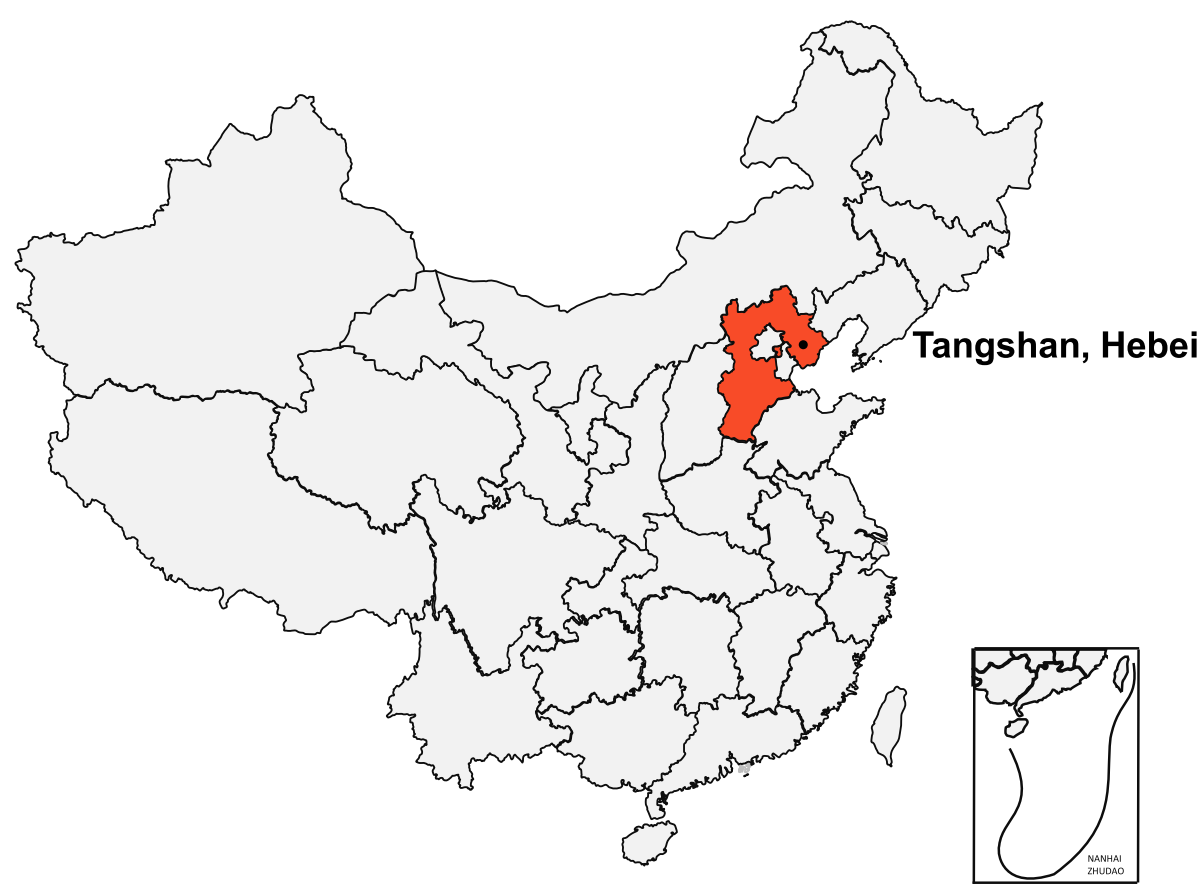

Fig. 1 The geographical location of participants in JECS 
the guidelines of the Declaration of Helsinki and was approved by the Ethics Committee of the Staff Hospital of the Jidong Oil-Field of Chinese National Petroleum (China National Petroleum Corporation Jidong Oil-Field Branch Staff Hospital approval document of the medical ethics committee, 2018 YILUNZI 1). All participants signed an informed consent form.

\section{Specific aims}

The primary objectives of this study were to clarify the pathogenesis of visual impairment from long-term follow-up observation in the Jidong community based on detailed etiological investigation, imaging and biomarkers, and establish a predictive model of visual impairment. The secondary objective of the JECS was to assess the association of CVD, including cerebrovascular disease, neurological disease, and other relevant disorders such as glucose metabolism and renal function, with ophthalmic disease.

\section{Participant enrollment}

The study consecutively recruited participants who had a physical examination in the Staff Hospital of Jidong Oil-Field of China National Petroleum Corporation (the employees and retirees of the Jidong Oil-Field of Chinese National Petroleum Company or their relatives) from the Jidong community who were age 18 years and older and had the ability to provide informed consent.

Participants with missing blood samples or an uncompleted questionnaire were excluded in this report. Additionally, we exclude participants who had CVD, neurological disease, a vision acuity lower than 0.1 logMAR and eye disease, or whose images, including those from fundus photography and OCT angiography, were of poor quality in the subsequent follow-up.

\section{Baseline data collection}

Baseline data were collected by the local clinicians and study personnel who were trained according to a standard protocol developed by the steering committee. The research investigators were trained by the field general office based on a standard protocol until they had the required capabilities. Research investigators enrolled consecutive participants, obtained informed consent and collected data by direct interviews.

A wide-ranging questionnaire comprised items such as demographics and lifestyle medical history. The demographics data included age, sex, marital status, occupation, income and education. The lifestyle data included items related to smoking, drinking, diet, exercise and the duration of nocturnal sleep. The medical history and current medication were also recorded. Women's health questions were asked of female participants. Mental state was assessed by the Mini Mental Status Examination
Scale (MMSE) and Montreal Cognitive Assessment (MoCA) [21-23]. The participants' height and weight were measured using a digital scale (in kilograms). The body mass index (BMI) was calculated by dividing the weight by the height squared. The waist-hip circumference was measured using a wall-mounted tape (in centimeters). Blood pressure and pulse were measured using a digital automatic blood pressure monitor after resting for five minutes. The blood pressure of the individual was then taken as the mean between the two closest readings. The blood pressure was estimated according to the American College of Cardiology/American Heart Association (ACC/AHA) Guideline [24].

An electronic data system combined with the paperbased questionnaire was developed for data collection. Trained research investigators stored the data on a laptop and then uploaded the data with their electronic signature later. All imaging examination and laboratory test results were uploaded to the electronic data system as pictures directly. All the data elements were checked manually by several independent study personnel in the field general office throughout the study period. We randomly checked the completed data and regularly monitored centrally to ensure the high quality of data collection. Additionally, an independent contract research organization will perform quality control of all the monitored items. All data elements collected were deidentified before analysis.

\section{Biological sample collection}

Food and drink were forbidden for $8 \mathrm{~h}$ before blood collection, and fasting elbow venous blood was collected by trained local clinicians in the morning and stored in vacuum tubes containing ethylenediaminetetraacetic acid and coagulation tubes. Hematological tests, such as fasting blood glucose, hemoglobin A1c (HBA1c), total cholesterol (TC), total triglycerides (TG), high-density lipoprotein (HDL-C), low-density lipoprotein (LDL-C), alanine aminotransferase, serum creatinine (Scr), and uric acid (UA) tests, were conducted first on fresh samples at the Central Laboratory of Jidong Oil-Field Hospital using an autoanalyzer (Hitachi, Tokyo, Japan). Additionally, blood samples were processed and the extracted serum, plasma and white blood cells were stored in the laboratory. Other biological samples, such as tongue coating samples, were also obtained. All the biological samples were stored in a $-80{ }^{\circ} \mathrm{C}$ freezer.

\section{Ophthalmic examinations}

The distance visual acuity (VA) of each eye (including uncorrected and corrected visual acuity) was measured using the Standard logarithmic VA Charts at a distance of $5 \mathrm{~m}$. If line 0.1 was not identified at $1 \mathrm{~m}$, the VA would be assessed by finger counting, hand movements, 
light perception, or no light perception along with the examination distance. We checked the right eye first followed by the left eye. Additionally, the refraction (including the diopter of sphere, diopter of column and axis) of the participant was measured using an autorefractor (KR800; Topcon Tokyo, Japan) to assess the participant's refractive status. The central corneal thickness $(\mathrm{CCT})$, aqueous depth (AD), lens thickness (LT), corneal curvature, pupil diameter and axial length (AL) were measured using optical low-coherence reflectometry (Lenstar 900 Optical Biometer; Haag-Streit, Koeniz, Switzerland). All participants were subjected to examinations of the anterior segments of the eye using a slitlamp microscope, the peripheral anterior chamber depth using the Van Herick method, and cataract using the Lens Opacities Classification System (LOCS III). The posterior segments were also examined using a slit-lamp microscope with a 90-diopter lens and mainly examined the optic disc, retinal vessels and macula. All participants had also undergone digital fundus photography (CR2AF; Canon Tokyo, Japan).

RTVue XR-100 with a central wavelength of $840 \mathrm{~nm}$ and a bandwidth of $45 \mathrm{~nm}$ (RTVue XR Avanti with AngioVue; Optovue Fremont, CA, USA) were used to measure retinal complex perfusion (RCP) and retinal thickness. The levels of the retinal superficial (the vessel between the internal limiting membrane and the inner plexiform layer) and deep (the vessel between the inner plexiform layer and the outer plexiform layer) vascular complex and choriocapillaris blood flow and flow void were measured using the optical coherence tomography angiography (OCTA) mode. An image quality score $\geq 6$ is considered qualified, otherwise it will be excluded. We analyzed the percent of blood flow and flow void in a certain area, which reflects the level of the blood perfusion in this sector. Additionally, choroidal thickness was measured using the Enhanced HD Line mode.

\section{Imaging data collection}

All participants were recommended for the following protocols: cardio-cerebrovascular examination: brain $\mathrm{CT}$, chest CT, resting 12-lead electrocardiogram (ECG) and rhythm strip recorded using a Cardio Perfect PCBased resting ECG system, and bilateral carotid duplex ultrasound; respiratory system examination: chest X-ray and obstructive spirometry; urinary system examination: B-ultrasonography and blood sample and urine assessments; female-specific characteristics: ultrasound, pelvic and gynecologic examinations and the Pap smear. All results are reviewed by two independent experts.

\section{Follow-up data collection and data management}

The follow-up period will be 5 years until December 31, 2024. All examinations will be performed by trained researchers or physicians designated by the hospital. Questionnaire, physical examination, ophthalmic examination and collection of biological samples will be conducted annually. Other examinations, such as intraocular pressure and visual field testing, will be tested in subsequent follow-ups. We record the occurrence and progression of visual impairment, collect cardio-cerebrovascular events, and assess neurological alternation such as cognitive impairment. For a detailed flow chart, see Fig. 2.

\section{Outcome measures}

The primary outcome is visual impairment. The level of visual impairment will be assessed by the visual acuity according to the WHO categories (1973) of low vision and blindness and some recent studies [25-28]. Visual impairment can be caused by diverse factors such as opacities of the refractive medium, amblyopia and, particularly, ocular vascular abnormality and optic nerve degeneration. The secondary outcomes include ocular vascular abnormality, optic nerve degeneration, CVD and cognitive impairment. We evaluated ocular vascular abnormality and optic nerve degeneration via comprehensive ocular assessment, particularly digital fundus photography and OCT. Ocular vascular abnormality refers to a series of pathological changes caused by diverse disorders. Additionally, optic nerve degeneration mainly presented as retinal specific layer alternation such as a nerve fiber layer defect (NFLD). All the images and data in this study are processed by two different experienced physicians; when a consensus on diagnosis cannot be reached, a third senior expert will determine the final diagnosis. CVD is diagnosed by physicians including a cardio-cerebrovascular specialist according to the International Classification of Diseases. It mainly includes nonfatal myocardial infarction, nonfatal heart failure, vascular-related operations and surgery, stroke (ischemic stroke, intracerebral hemorrhage, and subarachnoid hemorrhage), systemic embolism events, and cardiovascular death. Additionally, cognitive impairment will be assessed according to MMSE and MoCA.

\section{Statistical analysis}

Continuous variables are expressed as mean \pm standard deviation (SD) or median. Categorical variables are expressed as numbers with proportions. Student's t test or the Wilcoxon rank-sum test is used to compare the difference in the variables of interest between groups. The Chi-squared test is used to evaluate categorical variables. Multivariable analysis is used to evaluate associations between the parameters. The relevant statistical models are used to study the relationships between the variables of interest. Longitudinal analysis is performed to assess the relationship of annual risk factor changes 


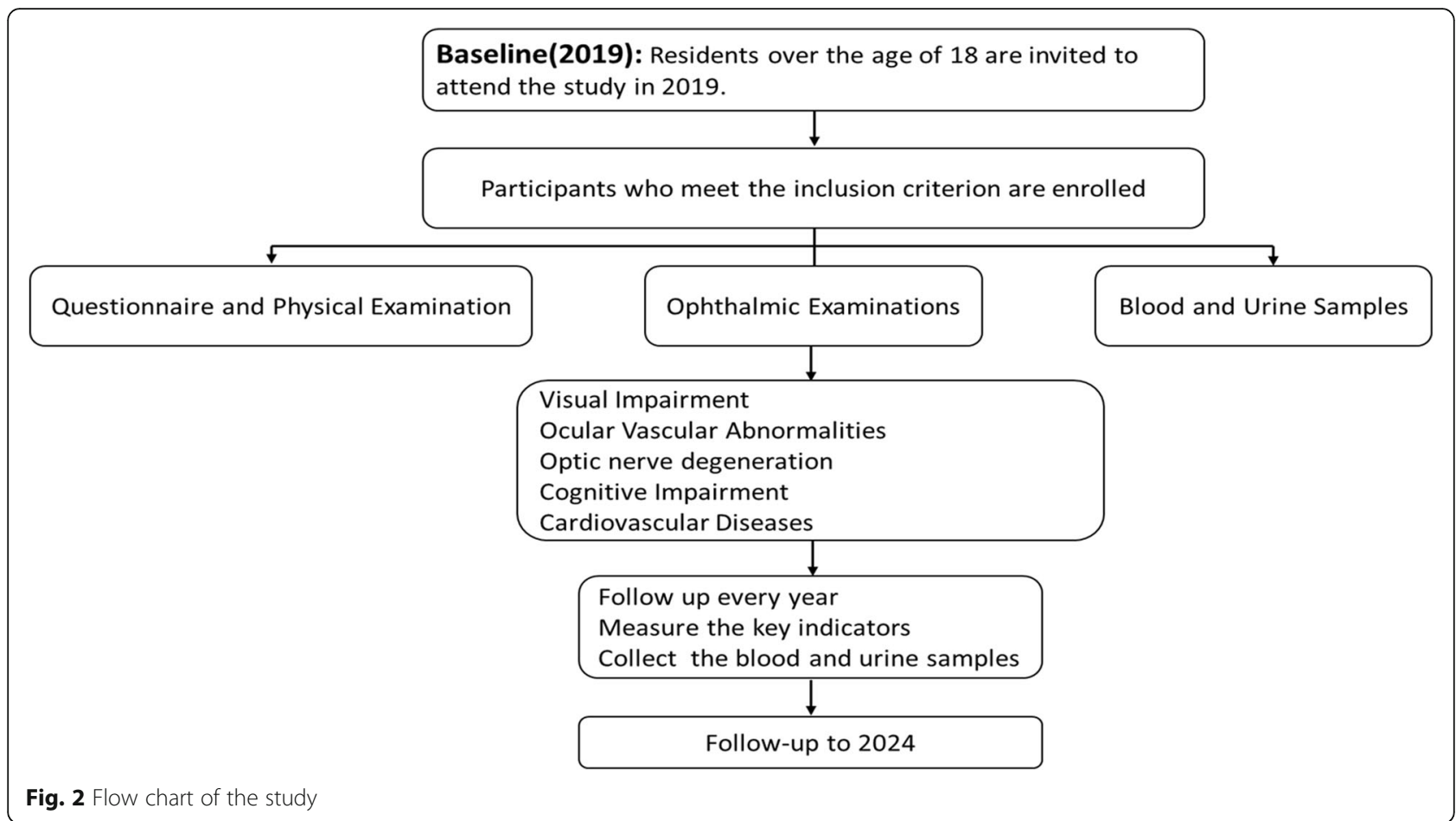

with the outcomes of interest. If necessary, multiple imputation is considered as a replacement for missing values. Statistical analysis is performed using SAS software (version 9.4; SAS Institute, Cary, NC, USA). The level of statistical significance is set at $\mathrm{P}<0.05$ (twotailed).

In this report, t-test and Chi-squared tests are used to compare the baseline characteristics between subjects grouped by sex and paired t-test for ocular characteristics between the right and left eyes.

\section{Results}

In total, 4,476 participants from the Jidong communities were initially screened and 1,099 participants among them were excluded due to missing blood samples or an uncompleted questionnaire (119 missing blood samples only, 867 have an uncompleted questionnaire, 113 both). Finally, 3,377 participants aged $45.0 \pm$ 12.5 years were enrolled between August 2019 and January 2020. The baseline characteristics of the included participants are summarized in Table 1. Overall, 53.6\% of these participants were women. Most (67.6\%) had completed college or higher, and $89.4 \%$ had a moderate income between $¥ 3000$ and $¥ 5000$ per month. Among the participants, $18.8 \%$ were current smokers and $13.6 \%$ were current drinkers. Overall, hypertension occurred in $825(25.0 \%)$, diabetes in 258 (7.7\%), hyperglycemia in $474(14.2 \%)$, and CVD history in $100(3.0 \%)$ participants.

Ocular characteristics of the participants are presented in Table 2. In this report, the right eye characteristics were stated only. For the right eye, the mean spherical equivalent refractive error was $-1.9 \pm 3.1$ diopters, with a mean $\mathrm{AL}$ of $24.2 \pm 1.4 \mathrm{~mm}$. The mean best-corrected visual acuity (BCVA) was $0.1 \log$ MAR in participants. The average OCT signal index was $8.2 \pm 1.2$. The Ganglion Cell Complex (GCC) thickness was $103.3 \pm 7.9 \mu \mathrm{m}$ for the entire measured area (0- to 3-mm diameter circle in the macula), while the whole macular thickness was $312.6 \pm 13.8 \mu \mathrm{m}$. The mean vessel densities were $46.4 \%$ and $50.8 \%$ for the superficial and deep vascular complexes, respectively. The mean area and perimeter of foveal avascular zone was $0.3 \mathrm{~mm}^{2}$ and $2.3 \mathrm{~mm}$, respectively.

\section{Discussion}

The JECS enrolled 3,377 participants from August 2019 to January 2020. The participant baseline characteristics and ocular characteristics were reported. Compared with several existing population-based cohorts, [29-37] the JECS is a unique and high-quality study focusing on ocular assessment along with comprehensive etiological evaluation, imaging and biomarker collection, providing the opportunity to identify risk factors, imaging and biomarkers of visual impairment (either ocular vascular anomalies or optic nerve degeneration) and to evaluate their associations with CVD and neurological diseases. Data from the JECS will provide evidence to formulate recommendations for optimal practice not only in visual impairment precaution but also in CVD and neurological diseases prevention (eye as a window) in subsequent phases. The findings 
Table 1 Baseline characteristics of eligible participants in the study

\begin{tabular}{|c|c|c|c|c|}
\hline Characteristics & Total & Male & Female & $P$ value \\
\hline $\bar{n}$ & 3377 & 1568 & 1809 & \\
\hline Age (SD), years & $45.0(12.5)$ & $45.1(12.5)$ & $44.9(12.6)$ & 0.61 \\
\hline Education level, n (\%) & & & & $<0.001$ \\
\hline Illiteracy / Primary School & $108(3.2)$ & $30(1.9)$ & $78(4.3)$ & \\
\hline Middle School & $978(29.2)$ & $405(26.0)$ & $573(31.9)$ & \\
\hline College / University & $2268(67.6)$ & $1122(72.1)$ & $1146(63.8)$ & \\
\hline Income, n (\%) & & & & $<0.001$ \\
\hline$<¥ 3,000$ & $284(8.5)$ & $91(5.8)$ & $193(10.8)$ & \\
\hline$¥ 3,000-5,000$ & $2999(89.4)$ & $1433(92.0)$ & $1566(87.2)$ & \\
\hline$>¥ 5,000$ & $70(2.1)$ & $34(2.2)$ & $36(2.0)$ & \\
\hline Current Smoking, n (\%) & $631(18.8)$ & $615(39.3)$ & $16(0.9)$ & $<0.001$ \\
\hline Current Drinking, n (\%) & $456(13.6)$ & $447(28.7)$ & $9(0.5)$ & $<0.001$ \\
\hline Hypertension, n (\%) & $825(25.0)$ & $531(34.3)$ & $294(16.8)$ & $<0.001$ \\
\hline Diabetes, n (\%) & $258(7.7)$ & $163(10.5)$ & $95(5.3)$ & $<0.001$ \\
\hline Hyperglycemia, n (\%) & $474(14.2)$ & $292(18.8)$ & $182(10.2)$ & $<0.001$ \\
\hline BMI $\left(\mathrm{kg} / \mathrm{m}^{2}\right)$ & $24.5 \pm 3.5$ & $25.6 \pm 3.2$ & $23.4 \pm 3.3$ & $<0.001$ \\
\hline Fasting Glucose (mmol/L) & $5.6 \pm 1.4$ & $5.8 \pm 1.6$ & $5.4 \pm 1.2$ & $<0.001$ \\
\hline Total Cholesterol (mmol/L) & $5.0 \pm 1.0$ & $5.0 \pm 1.0$ & $5.0 \pm 1.0$ & 0.781 \\
\hline Triglycerides (mmol/L) & $1.8 \pm 1.4$ & $2.1 \pm 1.6$ & $1.5 \pm 1.1$ & $<0.001$ \\
\hline MMSE score & $28.3 \pm 2.8$ & $28.3 \pm 2.7$ & $28.3 \pm 2.8$ & 0.96 \\
\hline MoCA score & $26.9 \pm 3.0$ & $26.9 \pm 3.0$ & $26.9 \pm 2.1$ & 0.997 \\
\hline CVD history, n (\%) & $100(3.0)$ & $50(3.2)$ & $50(2.8)$ & 0.532 \\
\hline
\end{tabular}

$B M I=$ body mass index,$M M S E=$ mini mental status examination scale, $M O C A=$ Montreal cognitive assessment, $C V D=$ cardiovascular disease

from the JECS will be updated to improve the clinical performance measures. The JECS would formulate the collaboration in clinical practice for ophthalmologists and cardiologists or neurologists. Vision health and general health of a person will be considered by ophthalmologists. Meanwhile, cardiologists and neurologists, will identify more alternatives for diagnosing CVD and neurological diseases from the eye.
The JECS has several strengths. First, a series of detailed ocular measurements is performed on participants, such as OCT angiography. In most cases, the small retina vessels and capillaries are first affected and pathologically changed due to CVD risk factors and various metabolic disorders [38, 39]. OCT angiography enables the visualization and quantitative analysis of retinal capillaries. Second, several relatively detailed cardio-cerebrovascular

Table 2 Ocular characteristics of eligible participants in the study

\begin{tabular}{|c|c|c|c|}
\hline Characteristics & OD & OS & Absolute Difference $(95 \% \mathrm{Cl})$ \\
\hline$\overline{B C V A}(\log M A R)$ & $0.1 \pm 0.1$ & $0.1 \pm 0.1$ & $0.0(0.0-0.0)$ \\
\hline SE (D) & $-1.9 \pm 3.1$ & $-1.8 \pm 2.9$ & $-0.1(-0.2-0.0)$ \\
\hline $\mathrm{AL}(\mathrm{mm})$ & $24.2 \pm 1.4$ & $24.2 \pm 1.4$ & $0.0(0.0-0.1)$ \\
\hline Signal Index & $8.2 \pm 1.2$ & $8.2 \pm 1.3$ & $0.0(0.0-0.0)$ \\
\hline Whole GCC thickness ( $\mu \mathrm{m})$ & $103.3 \pm 7.9$ & $103.2 \pm 7.8$ & $0.1(-0.1-0.3)$ \\
\hline Whole Macular thickness ( $\mu \mathrm{m})$ & $312.6 \pm 13.8$ & $312.7 \pm 13.8$ & $0.0(-0.5-0.4)$ \\
\hline Whole SCP Vessel Density (\%) & $46.4 \pm 3.2$ & $46.2 \pm 3.4$ & $0.0(-0.2-0.1)$ \\
\hline Whole DCP Vessel Density (\%) & $50.8 \pm 3.3$ & $51.0 \pm 3.6$ & $-0.3(-0.4--0.1)$ \\
\hline Foveal Avascular Zone Area $\left(\mathrm{mm}^{2}\right)$ & $0.3 \pm 0.2$ & $0.3 \pm 0.3$ & $0.0(0.0-0.0)$ \\
\hline Foveal Avascular Zone Perimeter (mm) & $2.3 \pm 0.6$ & $2.3 \pm 0.6$ & $0.0(0.0-0.0)$ \\
\hline
\end{tabular}

$B C V A=$ best-collect visual acuity, $S E=$ spherical equivalent, $A L=$ axial length, $G C C=$ ganglion cell complex, $S C P=$ superficial capillary plexus, $D C P=$ deep capillary plexus 
and neurological screening tests make it feasible to identify the association between vision impairment and CVD or cognitive impairment. The retinal vasculature shares many features with cardiovascular or cerebral vessels and is often exposed to the same environmental and intrinsic influences [40]. Therefore, the eye is a window to the heart or brain given its easily accessible vasculature. Additionally, certain unique vascular features in the retina are essential. Third, lifestyles of the participants in the JECS are collected through face-to-face interviews and followup and is performed annually. Finally, the longitudinal study will provide evidence to reveal the occurrence and development of ocular vascular abnormalities and optic nerve degeneration not only in ocular disease but also in cardio-cerebrovascular disease and cognitive impairment after several years of follow-up. The JECS contributes to the extensive evaluation of objective health conditions as well as environmental factors.

Our study also has several potential limitations. First, a selection bias may exist because the study was performed at a center where the economy is relatively developed and the population density is high. Moreover, some participants are recruited among the employees and retirees of the Jidong Oil-Field of Chinese National Petroleum Company who may not fully represent the entire Chinese population. Second, some of the data may be missing or subjects may be lost to follow-up due to the large number of examination items in this study. Considering this, we intensified the supervision to ensure the integrity of all the examination data. Additionally, the participants who would be followed up were notified in advance by telephone calls combined with text message reminders to reduce the rate of follow-up loss.

\section{Conclusions}

The JECS is a community-based, prospective, long-term follow-up observational study designed to identify the etiology, imaging and biomarkers for visual impairment and to evaluate their associations with risks of CVD and neurological diseases. The findings from the JECS will provide evidence for the risk factors of vascular abnormalities or neurodegeneration and further promote the establishment of early warning models of disease.

\footnotetext{
Abbreviations

JECS: Jidong Eye Cohort Study; OCT: Optical coherence tomography; CVD: Cardiovascular diseases; MMSE: Mini Mental Status Examination Scale; MoCA: Montreal Cognitive Assessment; BMI: Body mass index; ACC: American College of Cardiology; AHA: American Heart Association; HBA1c: Hemoglobin A1c; TC: Total cholesterol; TG: Total triglycerides; HDLC: High-density lipoprotein; LDL-C: Low-density lipoprotein; Scr: Serum creatinine; UA: Uric acid; VA: Visual acuity; CCT: Central corneal thickness; AD: Aqueous depth; LT: Lens thickness; AL: Axial length; LOCS III: Lens Opacities Classification System; RCP: Retinal complex perfusion; OCTA: Optical coherence tomography angiography; ECG: Electrocardiogram; NFLD: Nerve fiber layer defect; SD: Standard deviation; BCVA: Best-corrected visual acuity GCC: Ganglion cell complex
}

\section{Acknowledgements}

We are grateful to the Jidong community, Tangshan City, Hebei Province, China for its collaboration, especially the dedicated participants and all research staff involved in the study. We also thank Ruike Donghua

Translational Medical Research Center Co., Ltd. for supporting the project.

\section{Authors' contributions}

Study concept and design: JQ, FL, ML; data collection, analysis and interpretation: KY, LLC, XXZ, YFX, BBS, YZ, XYZ; manuscript writing: KY, GYZ; statistical analysis and administrative, technical or material support, and supervision: ML, DYS; critical revision of the manuscript for intellectual content: LLC, XWW. All authors approved the final version of the manuscript.

\section{Funding}

This study was supported by the National Natural Science Foundation of China, No. 81900903 (to ML); the Medical and Health Science and Technology Program of Zhejiang Province, No. $2017 \mathrm{KY} 113$ (to ML), $2018 \mathrm{KY} 543$ (to LLC). The funding bodies played no role in the study design, collection, analysis and interpretation of data.

\section{Availability of data and materials}

The datasets used and/or analyzed during the current study are available from the corresponding author upon reasonable request. The data that support the findings of this study are also available from Ruike Donghua Translational Medical Research Center.

\section{Ethics approval and consent to participate}

The study complies with the guidelines of the Declaration of Helsinki and was approved by the Ethics Committee of the Staff Hospital of the Jidong Oil-Field of Chinese National Petroleum (China National Petroleum Corporation Jidong Oil-Field Branch Staff Hospital approval document of the medical ethics committee, 2018 YILUNZI 1).

\section{Consent for publication}

The authors certify that they have obtained all appropriate participant consent forms. The participants understand that their names and initials will not be published and due efforts will be made to conceal their identity.

\section{Competing interests}

The authors declare that they have no competing interests.

\section{Author details}

${ }^{1}$ Eye Hospital and School of Ophthalmology and Optometry, Wenzhou Medical University, Wenzhou, Zhejiang, China. ${ }^{2}$ National Clinical Research Center for Ocular Diseases, Wenzhou, Zhejiang, China. ${ }^{3}$ Dezhou Center for Disease Control and Prevention, Dezhou, Shandong, China.

Received: 20 October 2020 Accepted: 23 November 2020

Published online: 29 December 2020

\section{References}

1. Bourne RRA, Flaxman SR, Braithwaite T, Cicinelli MV, Das A, Jonas JB, et al. Magnitude, temporal trends, and projections of the global prevalence of blindness and distance and near vision impairment: a systematic review and meta-analysis. Lancet Glob Health. 2017;5(9):e888-97.

2. Pascolini D. Mariotti SP. Global estimates of visual impairment: 2010. Br J Ophthalmol. 2012;96(5):614-8.

3. Xia F, Wu L, Weng C, Zhou X. Causes and three-year incidence of irreversible visual impairment in Jing-An District, Shanghai, China from 2010-2015. BMC Ophthalmol. 2017;17(1):216.

4. Chotikavanich S, Chanvarapha N, Loket S, Yingyong R, Dongngam S, Nujo W, et al. A 5-year retrospective record review of hospital-based low-vision rehabilitation in Thailand. Clin Optom (Auckl). 2018;10:41-50.

5. Ting DS, Cheung GC, Wong TY. Diabetic retinopathy: global prevalence, major risk factors, screening practices and public health challenges: a review. Clin Exp Ophthalmol. 2016;44(4):260-77.

6. Li JQ Welchowski T Schmid M, Letow J, Wolpers C, Pascual-Camps I, et al. Prevalence, incidence and future projection of diabetic eye disease in Europe: a systematic review and meta-analysis. Eur J Epidemiol. 2020:35(1): $11-23$. 
7. Colijn JM, Buitendijk GHS, Prokofyeva E, Alves D, Cachulo ML, Khawaja AP, et al. Prevalence of age-related macular degeneration in Europe: the past and the future. Ophthalmology. 2017;124(12):1753-63.

8. Fraser-Bell S, Symes R, Vaze A. Hypertensive eye disease: a review. Clin Exp Ophthalmol. 2017;45(1):45-53.

9. Iglicki M, Lavaque A, Ozimek M, Negri HP, Okada M, Chhablani J, et al. Biomarkers and predictors for functional and anatomic outcomes for small gauge pars plana vitrectomy and peeling of the internal limiting membrane in naive diabetic macular edema: The VITAL Study. PLoS ONE. 2018;13(7): e0200365.

10. Zur D, Iglicki M, Sala-Puigdollers A, Chhablani J, Lupidi M, Fraser-Bell S, et al. Disorganization of retinal inner layers as a biomarker in patients with diabetic macular oedema treated with dexamethasone implant. Acta Ophthalmol. 2020;98(2):e217-23.

11. Iglicki M, Busch C, Loewenstein A, Fung A, Invernizzi A, Mariussi M, et al. Underdiagnosed optic disk pit maculopathy: spectral domain optical coherence tomography features for accurate diagnosis. Retina. 2019;39(11): 2161-6.

12. GBD 2016 Risk Factors Collaborators. Global, regional, and national comparative risk assessment of 84 behavioural, environmental and occupational, and metabolic risks or clusters of risks, 1990-2016: a systematic analysis for the Global Burden of Disease Study 2016. Lancet. 2017;390(10100):1345-422.

13. GBD 2017 Risk Factor Collaborators. Global, regional, and national comparative risk assessment of 84 behavioural, environmental and occupational, and metabolic risks or clusters of risks for 195 countries and territories, 1990-2017: a systematic analysis for the Global Burden of Disease Study 2017. Lancet. 2018:392(10159):1923-94.

14. Murphy MP, Corriveau RA, Wilcock DM. Vascular contributions to cognitive impairment and dementia (VCID). Biochim Biophys Acta. 2016; 1862(5):857-9.

15. Corriveau RA, Bosetti F, Emr M, Gladman JT, Koenig Jl, Moy CS, et al. The science of vascular contributions to cognitive impairment and dementia (VCID): a framework for advancing research priorities in the cerebrovascular biology of cognitive decline. Cell Mol Neurobiol. 2016;36(2):281-8.

16. Patton N, Aslam T, Macgillivray T, Pattie A, Deary I, Dhillon B. Retinal vascular image analysis as a potential screening tool for cerebrovascular disease: a rationale based on homology between cerebral and retinal microvasculatures. J Anat. 2005;206(4):319-48.

17. Cheung $N$, Wong T. Diabetic retinopathy and systemic vascular complications. Prog Retin Eye Res. 2008;27(2):161-76.

18. Ong YT, Wong TY, Klein R, Klein BE, Mitchell $P$, Sharrett $A R$, et al. Hypertensive retinopathy and risk of stroke. Hypertension. 2013;62(4):70611.

19. Shantha GP, Srinivasan Y, Kumar AA, Salim S, Prabakhar S, Rajan AG, et al. Can retinal changes predict coronary artery disease in elderly hypertensive patients presenting with angina? Am J Emerg Med. 2010;28(5):617-21.

20. Khan Z, Almeida DRP, Rahim K, Belliveau MJ, Bona M, Gale J. 10-Year Framingham risk in patients with retinal vein occlusion: a systematic review and meta-analysis. Can J Ophthalmol. 2013;48(1):40-5.e1.

21. Lopez MN, Charter RA, Mostafavi B, Nibut LP, Smith WE. Psychometric properties of the Folstein Mini-Mental State Examination. Assessment. 2005; 12(2):137-44.

22. Patel M, Coshall C, Rudd AG, Wolfe CD. Natural history of cognitive impairment after stroke and factors associated with its recovery. Clin Rehabil. 2003;17(2):158-66.

23. Potocnik J, Ovcar Stante K, Rakusa M. The validity of the Montreal cognitive assessment (MoCA) for the screening of vascular cognitive impairment after ischemic stroke. Acta Neurol Belg. 2020;120(3):681-5.

24. Muntner P, Carey RM, Gidding S, Jones DW, Taler SJ, Wright JT Jr, et al. Potential U.S. population impact of the 2017 ACC/AHA High Blood Pressure Guideline. J Am Coll Cardiol. 2018;71(2):109-18.

25. Wei S, Sun Y, Li SM, Hu J, Cao K, Du J, et al. Visual impairment and spectacle use in university students in central China: the Anyang University Students Eye Study. Am J Ophthalmol. 2019;206:168-75

26. He M, Zeng J, Liu Y, Xu J, Pokharel GP, Ellwein LB. Refractive error and visual impairment in urban children in southern China. Invest Ophthalmol Vis Sci. 2004;45(3):793-9.

27. Zhao J, Ellwein LB, Cui H, Ge J, Guan H, Lv J, et al. Prevalence of vision impairment in older adults in rural China: the China Nine-Province Survey. Ophthalmology. 2010;117(3):409-16.e1.
28. Zhao J, Xu X, Ellwein LB, Cai N, Guan H, He M, et al. Prevalence of vision impairment in older adults in rural China in 2014 and comparisons with the 2006 China Nine-Province Survey. Am J Ophthalmol. 2018;185:81-93.

29. Liang YB, Friedman DS, Wong TY, Wang FH, Duan XR, Yang XH, et al. Rationale, design, methodology, and baseline data of a population-based study in rural China: the Handan Eye Study. Ophthalmic Epidemiol. 2009; 16(2):115-27.

30. Deng $Y$, Liang $Y$, Lin S, Wen L, Li J, Zhou Y, et al. Design and baseline data of a population-based metabonomics study of eye diseases in eastern China: the Yueqing Ocular Diseases Investigation. Eye Vis (Lond). 2020;7:8.

31. Chua SYL, Thomas D, Allen N, Lotery A, Desai P, Patel P, et al. Cohort profile: design and methods in the eye and vision consortium of UK Biobank. BMJ Open. 2019;9(2):e025077.

32. Meng Q, Cui Y, Zhang M, Zhang L, Zhang L, Zhang J, et al. Design and baseline characteristics of a population-based study of eye disease in southern Chinese people: the Dongguan Eye Study. Clin Exp Ophthalmol. 2016:44(3):170-80.

33. McKean-Cowdin R, Fairbrother-Crisp A, Torres M, Lastra C, Choudhury F, Jiang $X$, et al. The African American Eye Disease Study: design and methods. Ophthalmic Epidemiol. 2018;25(4):306-14.

34. Khanna RC, Murthy GV, Marmamula S, Mettla AL, Giridhar P, Banerjee S, et al. Longitudinal Andhra Pradesh Eye Disease Study: rationale, study design and research methodology. Clin Exp Ophthalmol. 2016;44(2):95-105.

35. Cooper LS, Wong TY, Klein R, Sharrett AR, Bryan RN, Hubbard LD, et al. Retinal microvascular abnormalities and MRI-defined subclinical cerebral infarction: the Atherosclerosis Risk in Communities Study. Stroke. 2006;37(1): 82-6.

36. Mitchell P, Wang JJ, Li W, Leeder SR, Smith W. Prevalence of asymptomatic retinal emboli in an Australian urban community. Stroke. 1997;28(1):63-6.

37. Wong TY, Klein R, Nieto FJ, Klein BEK, Sharrett AR, Meuer SM, et al. Retinal microvascular abnormalities and 10-year cardiovascular mortality: a population-based case-control study. Ophthalmology. 2003;110(5):933-40.

38. Østergaard L, Finnerup NB, Terkelsen AJ, Olesen RA, Drasbek KR, Knudsen L, et al. The effects of capillary dysfunction on oxygen and glucose extraction in diabetic neuropathy. Diabetologia. 2015:58(4):666-77.

39. Yu J, Gu R, Zong Y, Xu H, Wang X, Sun X, et al. Relationship between retinal perfusion and retinal thickness in healthy subjects: an optical coherence tomography angiography study. Invest Ophthalmol Vis Sci. 2016;57(9): OCT204-10.

40. Flammer J, Konieczka K, Bruno RM, Virdis A, Flammer AJ, Taddei S. The eye and the heart. Eur Heart J. 2013:34(17):1270-8.

Ready to submit your research? Choose BMC and benefit from:

- fast, convenient online submission

- thorough peer review by experienced researchers in your field

- rapid publication on acceptance

- support for research data, including large and complex data types

- gold Open Access which fosters wider collaboration and increased citations

- maximum visibility for your research: over $100 \mathrm{M}$ website views per year

At $\mathrm{BMC}$, research is always in progress.

Learn more biomedcentral.com/submissions 